\title{
Surface Treatments to Enhance the Functionality of PPEs
}

\author{
Pallab Sinha Mahapatra ${ }^{1} \odot$ - Souvick Chatterjee ${ }^{2} \cdot$ Manish K. Tiwari $^{3,4} \cdot$ Ranjan Ganguly $^{5} \cdot$ Constantine M. Megaridis $^{6}$
}

Received: 15 April 2020 / Revised: 14 May 2020 / Accepted: 19 May 2020 / Published online: 29 May 2020

(C) Indian National Academy of Engineering 2020

\begin{abstract}
The outbreak of unknown viral pneumonia in Wuhan China in December 2019 led to a new coronavirus (SARS-CoV-2), which attracted worldwide attention, with the related COVID-19 disease quickly becoming a global pandemic. In about 5 months, this disease has led to $\sim 4$ million cases and claimed more than $200 \mathrm{k}$ deaths as a result of its highly contagious nature. The present understanding is that SARS-CoV-2 is a type of influenza virus that can be transmitted through respiratory droplets and aerosols; Lewis (Nature 580:175, 2020). The primary methodology to prevent the spreading of this disease has been "social distancing" and usage of personal protective equipment (PPE) at the front lines of healthcare and other critical operations. The scale of the disease has led to unprecedented demand for PPEs and increased functionality of the same. This paper focuses on improving PPE functionality in a scalable manner by surface treatment and coating with appropriate materials and other functional enhancements, such as exposure to UV rays or other sterilizing agents (e.g., hydrogen peroxide).
\end{abstract}

Keywords Protective coatings $\cdot$ PPE $\cdot$ COVID-19 $\cdot$ SARS-CoV-2 $\cdot$ Nanocoatings

\section{Introduction}

Viral diseases frequently wreak havoc on public health by bringing inordinate numbers of patients to health care facilities within short time periods. The last 23 years have seen a rampage of several viral pandemics, like the severe acute respiratory syndrome coronavirus (SARS-CoV) in 2002-2003, the H1N1 influenza in 2009, the Middle East respiratory syndrome coronavirus (MERS-CoV) in 2012, and just recently, SARS-CoV-2 (Coronavirus disease 2019). This new virus appears to be highly contagious and has spread globally in record time. When the virus led to 118,000 cases in 114 countries and over 4000 deaths, the World Health Organization (WHO) declared COVID-19 a

Pallab Sinha Mahapatra

pallab@iitm.ac.in

Indian Institute of Technology Madras, Chennai, India

2 MathWorks India, New Delhi, India

3 Nanoengineered Systems Laboratory, UCL, London WC1E 7JE, UK

4 Wellcome/EPSRC Centre for Interventional and Surgical Sciences, UCL, London W1W 7TS, UK

5 Jadavpur University, Kolkata, West Bengal, India

6 University of Illinois at Chicago, Chicago, USA pandemic; Cascella et al. (2020). This novel virus is believed to have originated from the Wuhan district of China, and the first cases were linked to direct exposure traced to Huanan Seafood Wholesale Market of Wuhan, hinting towards the animal-human transmission (Chan et al. 2020). However, soon it was realized that the virus could be transmitted human-to-human, and symptomatic people became the primary source of COVID-19 spread. Like other respiratory pathogens, including flu and rhinovirus, the transmission is believed to occur through respiratory droplets from coughing, talking, and sneezing (Lewis 2020). Such respiratory droplets are $>5-10 \mu \mathrm{m}$ in diameter and are the primary source of transmission after a WHO study of 75,465 cases led to the dismissal of the possibility of airborne transmission. Van Doremalen et al. (2020) identified that aerosol transmission of SARS-CoV-2 is possible, and the virus may remain viable in the aerosol for hours. They also mentioned that SARS-CoV-2 could survive on different surfaces up to a few days.

The transmissibility of a virus is quantified by its basic reproduction rate $\left(R_{0}\right) ; R_{0}>1$ implies the number infected is likely to increase, whereas $R_{0}<1$ signifies transmission to die down gradually. Recent studies have shown that for COVID-19, the estimates of $R_{0}$ ranged from 1.4 to 6.49 , with a mean of 3.28 , a median of 2.79 , and interquartile range (IQR) of 1.16 (Liu et al. 2020). Countries like India, 
which has a population of 1.37 billion and a 2019 population growth rate of $1.08 \%$, are at high risk of such disease transmission. As a means of reducing such transmission, a critical component is the use of personal protective equipment (PPE), as recommended by WHO (2020a). In this work, we intend to provide an overview of coatings and treatments that can be used to enhance the functionality of existing PPEs or can provide antiviral properties, as demonstrated by other researchers as well as our research groups. To meet the high demand for PPEs with proper functionality in such a pandemic situation, we also review easy-to-use coating methodologies, which we have demonstrated earlier in unrelated studies.

As per the WHO guidelines, PPE is one of the most effective measures to avoid direct contamination from highly infectious pathogens, like SARS-CoV-2. The personal protection is critical for healthcare workers or any person who works in the high-risk zone of contagion. The complete PPE kit includes a face shield, goggles, mask, gloves, gowns/ coveralls, head and shoe covers (WHO 2020b; MoHFW 2020). These PPEs are also useful in protection from disease-causing microbes (e.g., viruses, bacteria, fungi, etc.) in general. Different components of PPE generally consist of different materials. For example, polycarbonate (PC) or cellulose acetate (CA) is typically used for the face shield, non-woven polypropylene fibers for masks, impermeable fabrics for shoe and headcovers. Whereas, gowns/coveralls are made up of materials that are impermeable to blood or other body fluids; MoHFW (2020).

\section{Antimicrobial Coatings}

Surface treatments to enhance resistance against diseasecausing microbes, i.e., antimicrobial coatings, have the potential to improve PPE functionalities dramatically. Over the last few decades, considerable research effort was made to incorporate antimicrobial coatings on the surfaces of various objects, such as garments and medical devices. Given the understandable concerns regarding drug resistance of these pathogens, commonly referred to as antimicrobial resistance (AMR), nanomaterial-based antimicrobial treatments are of particular interest. Bacteria have a very high tendency to develop AMR, whereby they become resistant to antibiotic drugs. Ravindra et al. (2010) fabricated antibacterial cotton fibers using silver nanoparticle coatings. The schematic of the coating process is shown in Fig. 1. They used $1 \mathrm{~mm}$-thick cotton fibers and immersed them in silver nitrate-containing leaf-extracted solution. The hydroxyl groups of the leaf extracts (polysaccharides) reduced the silver nitrate into silver nanoparticles on the cotton fibers. They used Gram-negative Escherichia coli (E. coli) bacteria to confirm the antibacterial activity of such cotton fibers and found excellent antibacterial properties of the cotton fibers even after several washing cycles. Such nanosilverbased materials may also provide antiviral features. Another complementary strategy comprises rendering the surfaces superhydrophobic. The repellence of the water droplets carrying the virus can give further improvement. The combined properties would hinder the penetration of respiratory droplets, thus facilitating virus containment.

\section{Hydrophobic Coatings on PPEs}

Hydrophobic coatings make it difficult for droplets/particles to adhere on surfaces and are known to provide antimicrobial characteristics, which are retained after multiple washes; antibacterial and antifungal properties were demonstrated by Mukherjee et al. (2008). Klibanov's group at MIT has shown extended functionality of hydrophobic coating characteristics against influenza viruses, which get transmitted through respiratory droplets, like SARS-CoV-2; Halder et al. (2008). The mechanism by which hydrophobization

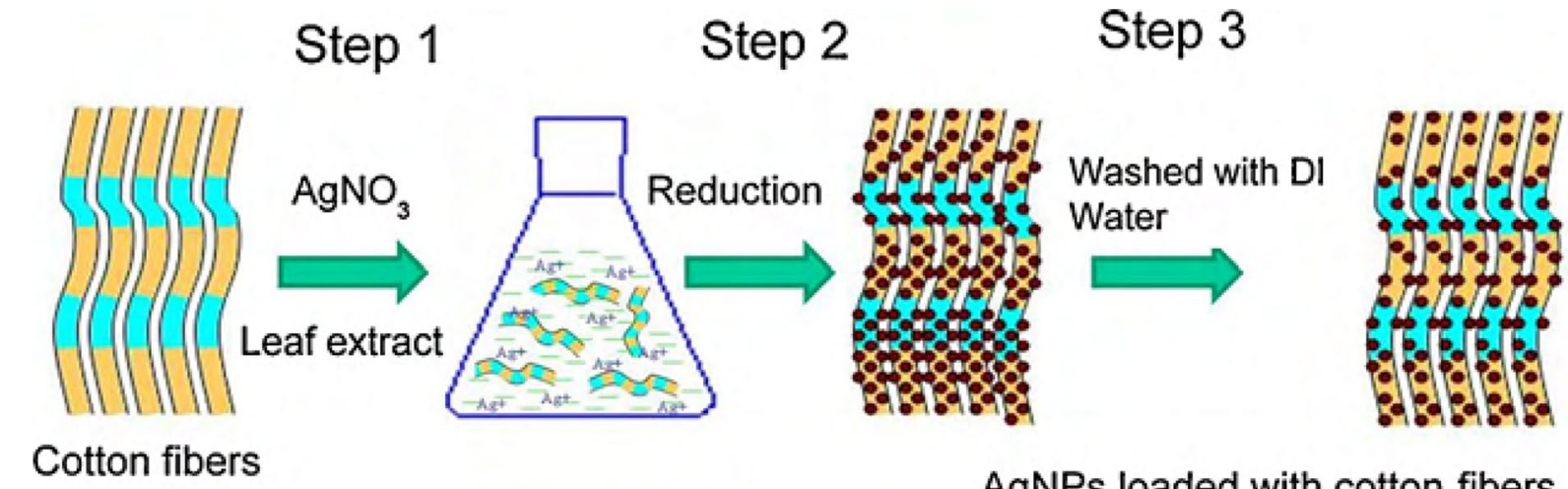

Step $1 \quad$ Step $2 \quad$ Step 3

Fig. 1 Antibacterial silver nanoparticle coating on cotton fibers. E. citriodora (Neelagiri) and F. bengalensis (Marri) leaf extract were used in the study of Ravindra et al. (2010) 
inactivates the virus has been described in detail by Hsu et al. (2011). Addressing the issue of creating hydrophobic coatings, Berendjchi et al. (2011) fabricated antibacterial cotton fabrics using copper nanoparticles and superhydrophobic coatings. They used a sol-gel method to make the cotton surface superhydrophobic.

Quan et al. (2017) used an evaporation-induced salt recrystallization method to create a virus inactivation system. They tested these salt-coated filters as a means against influenza viruses. Several nanoparticle-based coatings are also used as antimicrobial coatings. Compared to other nanoparticles like $\mathrm{Cu}, \mathrm{CuO}, \mathrm{Ag}, \mathrm{Si}, \mathrm{ZnO}_{2}$, etc., titanium dioxide $\left(\mathrm{TiO}_{2}\right)$ nanoparticles provide superior photocatalytic activities. It has been shown earlier that $\mathrm{TiO}_{2}$-coated surfaces have excellent antimicrobial properties; Wei et al. (2014). Due to photocatalytic activity, the antimicrobial performance of the $\mathrm{TiO}_{2}$-coated surfaces rises in the presence of sunlight; Wei et al. (2014). While typical UV (sunlight) is also known for its antibacterial properties, the photocatalytic activity of $\mathrm{TiO}_{2}$ is especially noteworthy because of its wettability characteristics in addition to its enhanced antibacterial and antiviral action. The photocatalytic properties of $\mathrm{TiO}_{2}$ have been exhaustively characterized by Nasikhuddin et al. (2018) who showed significant degradation of methylene blue on a $\mathrm{TiO}_{2}$ coating exposed to $\mathrm{UV}$, as compared to an identical control surface that was not exposed to UV.

In prior work, we have demonstrated a facile and scalable method of producing $\mathrm{TiO}_{2}$ nanoparticle coatings. We have examined coating performance on paper-based substrates (Chatterjee et al. 2018), non-woven materials (Sen et al. 2018) as well as polyethylene terephthalate (PET) substrates (Morrissette et al. 2017), or even by electrodeposition on a metal-wire mesh (Ghosh et al. 2020). As reported in Morrissette et al. (2017), to prepare a typical dispersion, we used $1 \mathrm{~g} \mathrm{TiO}_{2}$ nanoparticles ( $<25 \mathrm{~nm}$, Anatase; Sigma-Aldrich) in $13.25 \mathrm{~g}$ of ethanol (200 proof; Decan Labs). The solution was mixed by stirring and then probe-sonicated with $1000 \mathrm{~J}$ energy (750 W, $13 \mathrm{~mm}$ probe dia., $40 \%$ amplitude; Sonics \& Materials, Inc., Model VCX-750). $1.25 \mathrm{~g}$ of perfluoroalkyl methacrylate copolymer (PMC) $(20 \mathrm{wt} \%$ in water; DuPont, Capstone ST-100) was added to the $\mathrm{TiO}_{2}$-ethanol mixture, and the dispersion was left for $24 \mathrm{~h}$ to stabilize. After that, $10 \mathrm{~min}$ of bath sonication was required before spraying to the substrate. The substrate was heated for $2 \mathrm{~h}$ at $80^{\circ} \mathrm{C}$ to produce the micro/nano hierarchical superhydrophobic (contact angle $\theta_{\text {water }}^{*} \sim 160^{\circ} \pm 2^{\circ}$ ) coating. It is noted that upon UV exposure, the surface becomes more antimicrobial as well as less hydrophobic. Depending on the UV-exposure time, the wettability, as well as the antimicrobial properties, increases gradually. Spray or dip coating methods can alternatively be used to apply this coating on the PPE. The above methodology offers a simple, scalable procedure for producing antiviral coatings, to relieve the unprecedented demand for PPEs in the present time of distress. Additionally, it is crucial to have biodegradable PPEs to reduce impact on the environment. The coating methodology described earlier is biocompatible. During the pandemic situation, WHO has provided a guideline for reusing the disposable as well as reusable PPEs; WHO (2020a). Further, for reusing the PPEs prepared using the above methods, durability studies are required along with the identification of suitable binder polymers.

\section{Conclusion and Future Directions}

Scalable surface treatment strategies that combine antiviral action with liquid-repelling properties are one of many possible approaches to enhance the functionality of PPEs, thereby serving to satisfy their high demand in the healthcare industry and other fronts where the COVID-19 pandemic is being fought. We propose a low-cost coating approach that produces water-repellent coatings with additional sanitizing features due to the incorporation of a photocatalytic agent that is widely available commercially.

Acknowledgements We acknowledge the Scheme for Promotion of Academic and Research Collaboration (SPARC) program (Project: P644) from the Ministry of Human Resource Development, Government of India and the associated UKIERI support.

\section{References}

Berendjchi A, Khajavi R, Yazdanshenas ME (2011) Fabrication of superhydrophobic and antibacterial surface on cotton fabric by doped silica-based sols with nanoparticles of copper. Nanosc Res Lett 6:594

Cascella M, Rajnik M, Cuomo A, Dulebohn SC, Di Napoli R (2020) Features, evaluation and treatment coronavirus (COVID-19). StatPearls Publishing, Treasure Island

Chan JF, Yuan S, Kok KH, To KK, Chu H, Yang J, Xing F, Liu J, Yip CC, Poon RW, Tsoi HW (2020) A familial cluster of pneumonia associated with the 2019 novel coronavirus indicating person-to-person transmission: a study of a family cluster. Lancet 395:514-523

Chatterjee S, Sinha Mahapatra P, Ibrahim A, Ganguly R, Yu L, Dodge R, Megaridis CM (2018) Precise liquid transport on and through thin porous materials. Langmuir 34:2865-2875

Ghosh R, Sahu RP, Ganguly R, Zhitomirsky I, Puri IK (2020) Photocatalytic activity of electrophoretically deposited $\mathrm{TiO}_{2}$ and $\mathrm{ZnO}$ nanoparticles on fog harvesting meshes. Ceram Int 46:3777-3785

Haldar J, Chen J, Tumpey TM, Gubareva LV, Klibanov AM (2008) Hydrophobic polycationic coatings inactivate wild-type and zanamivir- and/or oseltamivir-resistant human and avian influenza viruses. Biotech Lett 30:475-479

Hsu BB, Wong SY, Hammond PT, Chen J, Klibanov AM (2011) Mechanism of inactivation of influenza viruses by immobilized hydrophobic polycations. Proc Natl Acad Sci 108:61-66

Lewis D (2020) Is the coronavirus airborne? Experts can't agree. Nature 580:175 
Liu Y, Gayle AA, Wilder-Smith A, Rocklöv J (2020) The reproductive number of COVID-19 is higher compared to SARS coronavirus. J Travel Med. https://doi.org/10.1093/jtm/taaa021

MoHFW (2020) https://www.mohfw.gov.in/. Accessed 11 Apr 2020

Morrissette JM, Sinha Mahapatra P, Ghosh A, Ganguly R, Megaridis CM (2017) Rapid, self-driven liquid mixing on open-surface microfluidic platforms. Sci Rep 7:1800

Mukherjee K, Rivera JJ, Klibanov AM (2008) Practical aspects of hydrophobic polycationic bactericidal "paints". Appl Biochem Biotechnol 151:61-70

Nasikhudin DM, Kusumaatmaja A, Triyana K (2018) Study on photocatalytic properties of $\mathrm{TiO}_{2}$ nanoparticle in various $\mathrm{pH}$ condition. J Phys Conf Ser 1011:012069

Quan FS, Rubino I, Lee S-H, Koch B, Choi H-J (2017) Universal and reusable virus deactivation system for respiratory protection. Sci Rep 7:39956

Ravindra S, Murali Mohan Y, Narayana Reddy N, Mohana Raju K (2010) Fabrication of antibacterial cotton fibres loaded with silver nanoparticles via "Green Approach". Colloids Surf A 367:31-40

Sen U, Chatterjee S, Sinha Mahapatra P, Ganguly R, Dodge R, Yu L, Megaridis CM (2018) Surface-wettability patterning for distributing high-momentum water jets on porous polymeric substrates. ACS Appl Mater Interfaces 10:5038-5049 van Doremalen N, Bushmaker T, Morris DH, Holbrook MG, Gamble A, Williamson BN, Tamin A, Harcourt JL, Thornburg NJ, Gerber SI, Lloyd-Smith JO, de Wit E, Munster VJ (2020) Aerosol and surface stability of SARS-CoV-2 as compared with SARS-CoV-1. N Engl J Med 382:1564-1567

Wei X, Yang Z, Tay SL, Gao W (2014) Photocatalytic $\mathrm{TiO}_{2}$ nanoparticles enhanced polymer antimicrobial coating. Appl Surf Sci 290:274-279

World Health Organization (2020) Rational use of personal protective equipment for coronavirus disease (COVID-19) and considerations during severe shortages. https://www.who.int/publicationsdetail/rational-use-of-personal-protective-equipment-for-coron avirus-disease-(covid-19)-and-considerations-during-severe-short ages. Accessed 11 Apr 2020

World Health Organization (2020) Personal protective equipment. WHO https://www.who.int/medical_devices/meddev_ppe/en/. Accessed 13 Apr 2020

Publisher's Note Springer Nature remains neutral with regard to jurisdictional claims in published maps and institutional affiliations. 